KANSAS JOURNAL of MEDICINE

\section{Rare Association of Absent Pulmonary Valve Syndrome with Double Outlet Right Ventricle and Hypoplastic Left Heart Complex}

Erik L. Frandsen, M.D. ${ }^{1}$, Arpan R. Doshi, M.D. ${ }^{2}$,

Sujatha Buddhe, M.D. ${ }^{1}$, Bhawna Arya, M.D. ${ }^{1}$,

Sathish M. Chikkabyrappa, M.D. ${ }^{1}$

'Seattle Children's Hospital and School of Medicine,

University of Washington, Seattle, WA

${ }^{2}$ Children's Cardiology Associates, Mednax Health Solutions Partner, Houston, TX

Received Jun. 21, 2019; Accepted for publication Aug. 27, 2019; Published online Jan. 31, 2020

\section{INTRODUCTION}

Absent pulmonary valve (APV) syndrome is rare congenital cardiac anomaly and usually associated with Tetralogy of Fallot (TOF) in about $3 \%$ to $6 \%$ of cases. ${ }^{1}$ A combination of double outlet right ventricle (DORV) and APV syndrome has been described in the literature. A few case reports of APV syndrome with single ventricle physiology like tricuspid atresia and pulmonary atresia with intact ventricular septum have been described and successfully palliated., ${ }^{2,3}$ To our knowledge, APV syndrome has not been described in association with hypoplastic left heart in the context of DORV. Such patients with conotruncal anomalies have genetic predisposition for $22 \mathrm{q} 11.2$ deletion (DiGeorge syndrome).

DORV with associated remote ventricular septal defect (VSD), APV syndrome, and hypoplastic left ventricle is an extremely rare variation of DORV. Patients with APV syndrome have a dysplastic or absent pulmonary valve, annular stenosis, and free pulmonary insufficiency. It is associated with aneurysmal main and branch pulmonary arteries that often compromises respiratory function due to mechanical airway compression. ${ }^{4}$ We describe this rare variation of DORV with remote VSD, absent pulmonary valve, and hypoplastic left ventricle.

\section{CASE REPORT}

A 23-year-old pregnant Caucasian woman was referred for fetal echocardiogram at 20 weeks gestation due to concerns of congenital heart disease on obstetric anatomy scan. The remainder of the maternal medical and family history was unremarkable. The fetal echocardiogram demonstrated DORV with normally related great arteries, remote VSD, and moderate to severe hypoplastic mitral valve and left ventricle. Pulmonary artery architecture was suggestive of APV with to-and-fro flow across the dysplastic and rudimentary pulmonary valve tissue. There was a moderate degree of stenosis with the peak velocity of $3.0 \mathrm{~m} / \mathrm{s}$ across the rudimentary pulmonary valve tissue. The main and branch pulmonary arteries were severely dilated. Right ventricular systolic function was below normal. There was no evidence of ductus arteriosus (Figure 1). The family was counseled regarding this complex diagnosis and poor prognosis.

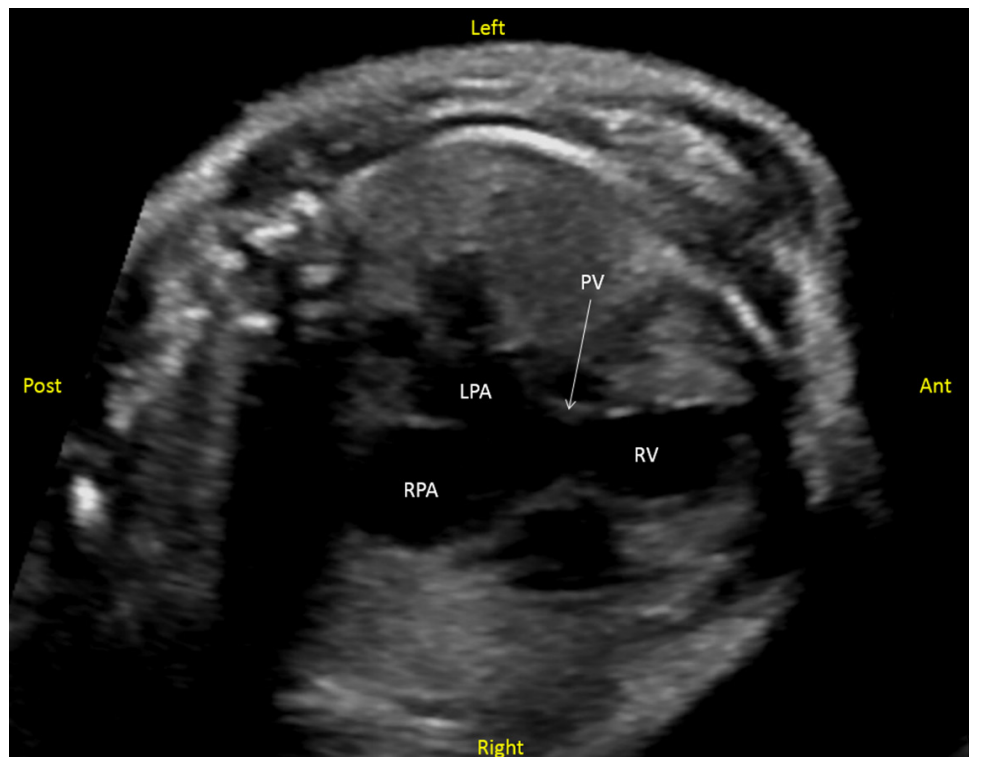

Figure 1. Transverse axial 2-D still frame of a fetal echocardiogram demonstrated the absent pulmonary valve with severely dilated main and branch pulmonary arteries. PV: pulmonary valve; LPA: left pulmonary artery; RPA: right pulmonary artery; $\mathrm{RV}$ : right ventricle.

The pregnancy was complicated by polyhydramnios which was treated with amnioreduction. Genetic analysis of amniotic fluid detected 22q11.2 deletion, consistent with DiGeorge syndrome. The baby was delivered at 36 weeks gestation via cesarean section due to breech position and premature rupture of membranes from severe polyhydramnios. The baby's birth weight was 2300 grams.

The patient was cyanotic and apneic at birth requiring intubation with an initial blood gas showing pH 7.17, pCO2 69, pO2 21 with oxygen saturations of $70 \%$. Upon admission to the cardiovascular intensive care unit, the baby continued to be hypoxic on $100 \%$ fractional inspired oxygen, inhaled nitric oxide and prone positioning. Physical exam revealed a profoundly hypoxic and cyanotic female neonate with dysmorphic features consistent with 22q.11 deletion. Cardiac auscultation was notable for a to-and-fro murmur along the left sternal border.

The initial postnatal transthoracic echocardiogram revealed DORV with sub-aortic VSD and side-by-side great arteries (aortic valve rightward to the pulmonary valve) with aortic-mitral fibrous discontinuity. The mitral valve was severely hypoplastic (z-score -5.1) with minimal antegrade flow and the left ventricle was hypoplastic and non-apex forming (Figure 2). Aortic valve leaflets were thickened and dysplastic with normal annular measurements. The valve annulus measured $0.9 \mathrm{~cm}(\mathrm{z}$-score +3.2$)$ and aortic sinuses measured $1.22 \mathrm{~cm}(\mathrm{z}$-score +3$)$. There was no evidence of coarctation of aorta. Aortic isthmus measured $0.4 \mathrm{~cm}$ (z-score -1.2) which was within the normal range. Pulmonary valve leaflets were dysplastic and rudimentary (consistent with APV syndrome) with mild valvar stenosis and severe/free insufficiency with to-and-fro flow on color Doppler. The main pulmonary artery was severely dilated at $13 \mathrm{~mm}(\mathrm{z}$-score +5.42$)$ with severely dilated branch pulmonary arteries. The left pulmonary artery (LPA) measured $7.5 \mathrm{~mm}(\mathrm{z}$-score + 3.99) and the right pulmonary artery (RPA) measured $8 \mathrm{~mm}$ in size (z- score +4.21 ; Figure 3). The right ventricle was dilated and hypertrophied with severely depressed systolic function (fractional shortening 19\%). There was a partially restrictive atrial septum with left to right shunting. 


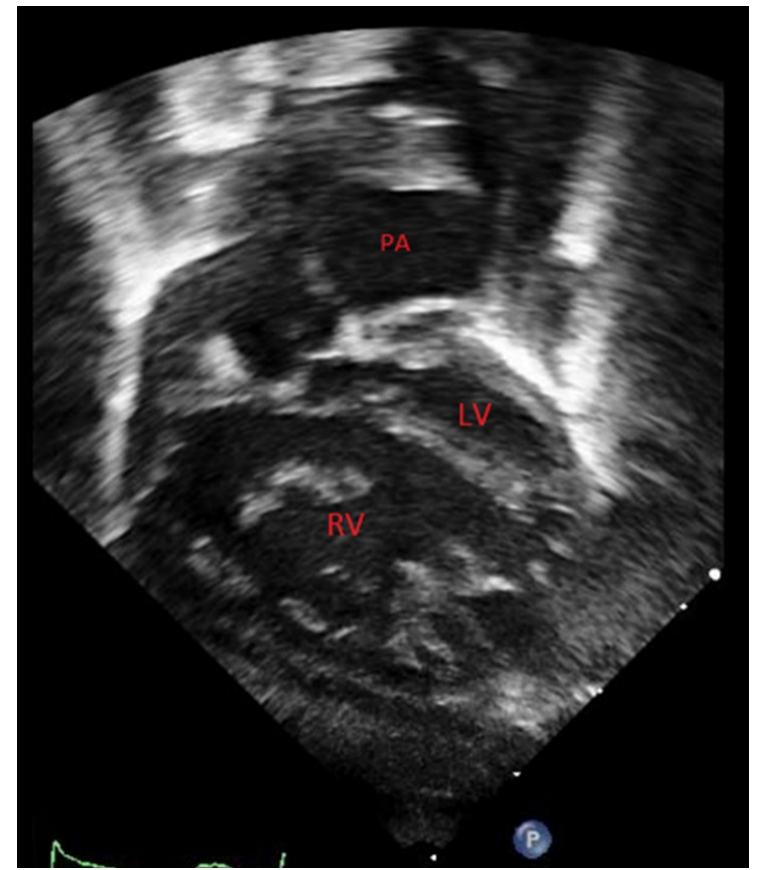

Figure 2. Transthoracic echocardiogram in apical view demonstrated severely hypoplastic non-apex forming left ventricle (LV), dilated right ventricle (RV) and severely dilated pulmonary artery (PA).

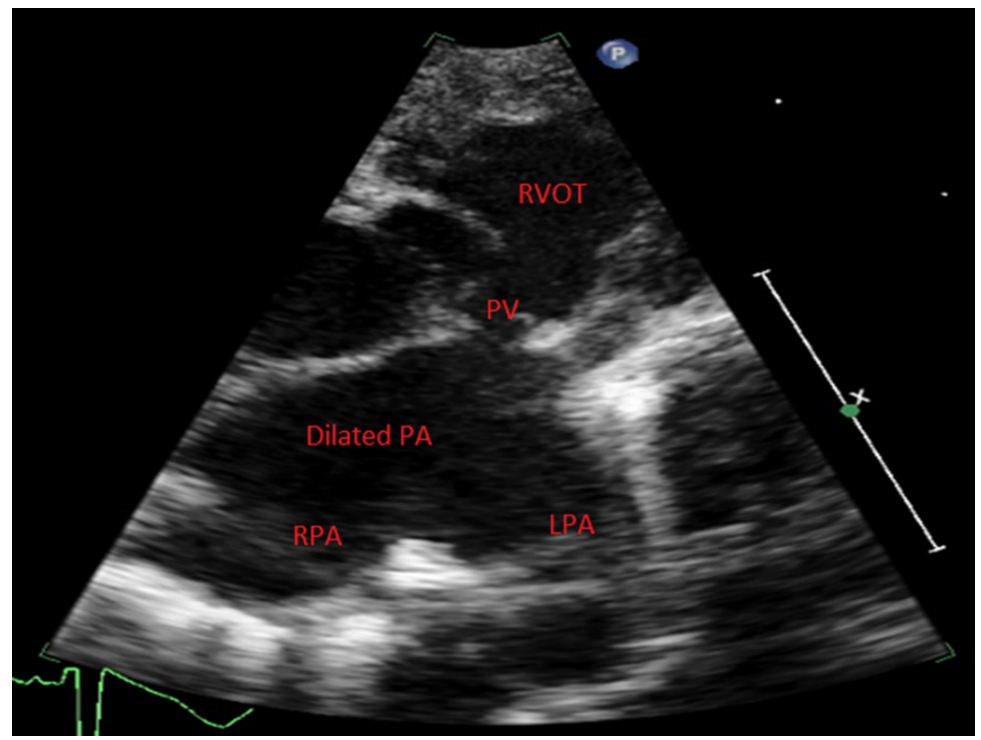

Figure 3. Parasternal short axis view of transthoracic echocardiogram demonstrating severely dilated aneurysmal main and branch pulmonary arteries and dysplastic and rudimentary pulmonary valve. RPA - right pulmonary artery; LPA - left pulmonary artery; RVOT - right ventricular outflow tract; PV - pulmonary valve; PA - pulmonary artery.

Due to refractory cardiogenic shock with severe hypoxemia, the decision was made to proceed with surgical intervention within the first 12 hours of life. The surgery consisted of atrial septectomy, branch pulmonary artery plication, over sewing of the main pulmonary artery, and placement of a right $3.5 \mathrm{~mm}$ modified BlalockTaussig-Thomas shunt to achieve controlled pulmonary blood flow. Unfortunately, the patient failed to separate from cardiopulmonary bypass due to ongoing severe ventricular dysfunction and was transitioned to extracorporeal membrane oxygenator support.

The immediate postoperative course was complicated by hemothorax and acute kidney injury requiring continuous renal replacement therapy. She was profoundly immunocompromised with severe lymphopenia and hypogammaglobinemia secondary to DiGeorge
KANSAS JOURNAL of MEDICINE ABSENT PULMONARY VALVE SYNDROME continued.

syndrome. She developed septic shock from Serratia marcescens septicemia and abdominal compartment syndrome on post-operative day 24, requiring abdominal decompression and washout. She continued to deteriorate hemodynamically, with development of multi-organ failure, including profound neurological impairment. Continued detailed discussions between the clinical team and family surrounding goals of care and prognosis in the setting of multi-organ failure, led to the decision of comfort care. The patient died on postoperative day 31 .

\section{DISCUSSION}

APV syndrome usually is associated with other congenital heart defects, notably TOF. We reported a rare association of APV syndrome with a hypoplastic left heart variant in the setting of DORV with side-by-side great vessels. Abnormal pulmonary vasculature and elevated pulmonary vascular resistance in the setting of single ventricle physiology makes single ventricle palliation very challenging and these patients are usually not good candidates for Fontan palliation. Additional association of genetic mutations further complicates the clinical course. Detailed prenatal counselling regarding the potential grave prognosis and multidisciplinary approach to care and decisionmaking is imperative.

\section{CONCLUSION}

APV in association with single ventricle anatomy carries a poor prognosis. Prenatal genetic testing should be considered in these cases to aid further risk assessment, as presence of genetic abnormalities further worsens the prognosis. It is highly recommended to consider delivering the fetus at a tertiary level pediatric cardiac center as the baby is likely to need intensive cardiac interventions soon after birth.

\section{REFERENCES}

${ }^{1}$ Bharati AH, Naware A, Merchant SA. Absent pulmonary valve syndrome with tetralogy of Fallot and associated dextrocardia detected at an early gestational age of 26 weeks. Indian J Radiol Imaging 2008; 18(4):352-354. PMID: 19774197.

${ }^{2}$ Litovsky S, Choy M, Park J, at al. Absent pulmonary valve with tricuspid atresia or severe tricuspid stenosis: Report of three cases and review of the literature. Pediatr Dev Pathol 2000; 3(4):353-366. PMID: 10890251.

Forrest P, Bini RM, Wilkinson JL, et al. Congenital absence of the pulmonic valve and tricuspid valve atresia with intact ventricular septum. Am J Cardiol 1987; 59(5):482-484. PMID: 3812322.

4 Zucker N, Rozin I, Levitas A, Zalzstein E. Clinical presentation, natural history, and outcome of patients with the absent pulmonary valve syndrome. Cardiol Young 2004; 14(4):402-408. PMID: 15680047.

Keywords: double outlet right ventricle, pulmonary valve, hypoplastic left heart syndrome, ventricular heart septal defects 